\title{
Guaranteed and randomized methods for stability analysis of uncertain metabolic networks
}

\author{
Heinz Koeppl, Stefano Andreozzi and Ralf Steuer
}

\begin{abstract}
A persistent problem hampering our understanding of the dynamics of large-scale metabolic networks is the lack of experimentally determined kinetic parameters that are necessary to build computational models of biochemical processes. To overcome some of the limitations imposed by absent or incomplete kinetic data, structural kinetic modeling (SKM) was proposed recently as an intermediate approach between stoichiometric analysis and a full kinetic description. SKM extends stationary flux-balance analysis (FBA) by a local stability analysis utilizing an appropriate parametrization of the Jacobian matrix. To characterize the Jacobian, we utilize results from robust control theory to determine subintervals of the Jacobian' entries that correspond to asymptotically stable metabolic states. Furthermore, we propose an efficient sampling scheme in combination with methods from computational geometry to sketch the stability region. A glycolytic pathway model comprising 12 uncertain parameters is used to assess the feasibility of the method.
\end{abstract}

\section{Modeling metabolic networks}

Cellular metabolism, defined as the orchestrated biochemical interconversion of small molecules by dedicated proteins, is an important aspect of cellular physiology and of outstanding interest for many biotechnological and medical applications. In the past decades, great strides have been made to elucidate and compile the list of the biochemical reaction taking place in living cells and almost comprehensive sto-

Heinz Koeppl and Stefano Andreozzi

Laboratory of Nonlinear Systems, School of Communication and Computer Sciences, Ecole Polytechnique Federale de Lausanne (EPFL), 1015 Lausanne, Switzerland e-mail: \{heinz.koeppl, stefano.andreozzi\}@epfl.ch

Ralf Steuer

Institute for Theoretical Biology, Humboldt University of Berlin, Invalidenstrasse 43, D-10115 Berlin, Germany and Manchester Interdisciplinary Biocentre, The University of Manchester, Manchester M1 7DN, UK. e-mail: ralf.steuer@manchester.ac.uk 
ichiometric models for several model organisms such as the S. cerevisiae or E. coli, are now available [10]. However, to obtain a true understanding of cellular function and organization a mere list of parts is not enough. In this respect, the construction of mathematical models is an indispensable tool to study - and eventually understand - how the parts of a metabolic network interact and function as an integrated whole.

Current approaches to metabolic modeling are characterized by a dichotomy of large-scale constraint-based stoichiometric models on the one hand, and detailed kinetic models of small subsystems on the other hand. The advantage of topological and constraint-based methods is that they only require stoichiometric information, making them applicable to large, up to 'genome-scale', systems. Most prominently, flux-balance analysis (FBA) makes use of the mass conservation constraints to identify possible flux distributions that fulfill a given objective function, such as maximal ATP production or maximal biomass generation. Although one of the most successful approaches to date, the downside of FBA is that it cannot provide any information about the dynamical properties of the metabolic system. In contrast, the description of dynamics requires the construction of a detailed kinetic model of the network, usually in terms of ordinary differential equations. However, the construction of such explicit kinetic models of metabolism is based on detailed quantitative information on kinetic parameters and rate equations, information that is only rarely available in practice.

To overcome some of the difficulties imposed by the lack of information on kinetic parameters, there has been increasing interest in heuristic and semi-quantitative methods to describe the dynamics of large-scale metabolic networks in the face of uncertain kinetic data [23, 22, 20]. Specifically, structural kinetic modeling (SKM) proposes to augment the constraint-based analysis by a local stability analysis utilizing an appropriate parametrization of the Jacobian matrix [21]. The approach is based on the observation that in many cases a detailed kinetic model is not necessary. Rather, a large number of dynamical properties, such as control coefficients, the stability of states, transitions to oscillatory regions, among various others, are readily available using only a linear approximation of the system. SKM therefore seeks to derive stringent bounds on the entries of the Jacobian matrix, based on available phenotypic data and biophysical constraints, to enable a computational analysis in the absence of further kinetic information. We emphasize that SKM is a data-driven approach, taking another perspective than classical nonlinear dynamics. More specifically, SKM starts out with a given, experimentally measured steady state and asks for the underlying parameter region supporting this particular state.

In this work, we discuss an extension of SKM utilizing methods from robust stability theory $[4,1]$ that allows to determine subintervals of the Jacobian entries of a SKM model corresponding to stable metabolic states. To this end, we believe that the proposed reasoning about entire sets of models is an adequate semi-quantitative approach [18] to analyze biochemical models in general.

The paper is organized as follows. Section 2 provides an introduction to the SKM framework. The applied guaranteed methods from robust control as well as a novel random sampling scheme are discussed in Section 3. An application of the sampling method is given in Section 4, while Section 5 draws conclusions. 


\section{Structural kinetic modeling}

SKM draws upon the fact that, even in the absence of detailed kinetic information, questions with respect to stability of the metabolic operating point can be addressed. To this end, we consider a metabolic network whose time-dependent behavior is described by an ordinary differential equation of the form

$$
\frac{\mathrm{d} \mathbf{S}}{\mathrm{d} t} \equiv \dot{\mathbf{S}}=\mathbf{N v}(\mathbf{S}, \mathbf{k})
$$

with $\mathbf{S} \in \mathbb{R}_{+}^{N}$ denoting the vector of concentration of all involved species, $\mathbf{N} \in \mathbb{Z}^{N \times L}$ the stoichiometric matrix, $\mathbf{v}: \mathbb{R}_{+}^{N} \times \mathbb{R}_{+}^{M} \rightarrow \mathbb{R}_{+}^{L}$ the parametric rate laws and $\mathbf{k} \in \mathbb{R}_{+}^{M}$ a vector comprising all kinetic parameters. We assume that the network has at least one non-zero steady state at concentration $\mathbf{S}^{0}$, which does not necessarily has to be stable. In this case, we can equivalently write

$$
\frac{\dot{S}_{i}}{S_{i}^{0}}=\sum_{j=1}^{R} N_{i j} \frac{v_{j}\left(\mathbf{S}^{0}\right)}{S_{i}^{0}} \frac{v_{j}(\mathbf{S})}{v_{j}\left(\mathbf{S}^{0}\right)} .
$$

Introducing concentrations that are normalized by the steady state concentration $x_{i}=\frac{S_{i}}{S_{i}^{0}}$ one obtains

$$
\dot{\mathbf{x}}=\boldsymbol{\Lambda} \boldsymbol{\mu}(\mathbf{x}),
$$

with the constant matrix $\Lambda_{i j} \equiv N_{i j} \frac{{ }^{{ }_{j}}\left(\mathbf{S}^{0}\right)}{S_{i}^{0}}$ and the vector of normalized fluxes $\mu_{j}(\mathbf{x}) \equiv$ $\frac{v_{j}(\mathbf{S})}{v_{j}\left(\mathbf{S}^{0}\right)}$. A linearization of the system at the steady state $\mathbf{x}=\mathbf{1}$ yields with $\boldsymbol{\Lambda} \boldsymbol{\mu}(\mathbf{1})=\mathbf{0}$ a linear model with states $z_{i}$

$$
\dot{z}_{i}=\left.\sum_{j=1}^{L} \sum_{k=1}^{N} \Lambda_{i j} \frac{\partial \mu_{j}(\mathbf{z})}{\partial z_{k}}\right|_{\mathbf{z}=\mathbf{1}}\left(z_{k}-1\right)
$$

Introducing the matrix $\left.\Theta_{k j} \equiv \frac{\partial \mu_{j}(\mathbf{z})}{\partial z_{k}}\right|_{\mathbf{z}=\mathbf{1}}$ we obtain

$$
\dot{\mathbf{z}}=\boldsymbol{\Lambda} \Theta(\mathbf{z}-\mathbf{1}) .
$$

The stability of the nonlinear system specified by (3) at $\mathbf{x}=\mathbf{1}$ is thus determined by the eigenvalues of the matrix $\Lambda \Theta$, which is equivalent to the (scaled) Jacobian matrix. Our further analysis rests upon a detailed interpretation of the matrices $\boldsymbol{\Lambda}$ and $\boldsymbol{\Theta}$. In particular, the matrix $\boldsymbol{\Lambda}$ is entirely specified by stoichiometric information, along with knowledge of a stationary metabolic state, characterized by a set of stationary concentrations $\mathbf{S}^{0}$ and fluxes $\mathbf{v}^{0}=\mathbf{v}\left(\mathbf{S}^{0}\right)$. The latter satisfy the steady-state constraint $\mathbf{N} \mathbf{v}^{0}=\mathbf{0}$. We note that large-scale measurements and the characterization of metabolic systems in terms of concentrations (metabolomics) and fluxes (fluxomics) are now almost standard techniques in the analysis of cellular 
metabolism [14, 24, 17], making the matrix $\boldsymbol{\Lambda}$ - at least in principle - accessible to direct experimentation.

The interpretation of the elements of $\Theta$ is slightly more intricate. Every entry of the matrix $\Theta$ specifies the derivatives of the normalized rate law with respect to the scaled concentrations, and can be interpreted as the (dimensionless) relative saturation level of one particular reaction with respect to one particular substrate concentration. Importantly, for most typical rate laws the elements of $\Theta$ are confined to well-defined intervals that are independent of the respective metabolic state or mathematical details of the rate equation. We note that the elements of $\boldsymbol{\Theta}$ are analogous to logarithmic derivatives and are closely related to the scaled elasticity coefficients in Metabolic Control Analysis [12].

\section{Stability of uncertain linear systems}

We are now in the position to apply the ideas of robustness analysis for linear systems to the Jacobian matrix $\mathbf{J} \equiv \boldsymbol{\Lambda} \boldsymbol{\Theta}$ of our linearized metabolic network. Allowing uncertainty in the kinetic rate law corresponds here to an uncertainty about the saturation matrix $\Theta$. Thus we define the set of Jacobians as

$$
\mathbf{J}([\boldsymbol{\Theta}])=\left\{\mathbf{J} \mid \mathbf{J}=\boldsymbol{\Lambda} \boldsymbol{\Theta}, \boldsymbol{\Theta} \in[\boldsymbol{\Theta}] \in \mathbb{\mathbb { R } ^ { L \times N }}\right\},
$$

where is $\mathbb{I}$ is the set of all real intervals. Thus an element $[\Theta] \in \mathbb{I} \mathbb{R}^{N \times L}$ is an interval matrix

$$
[\boldsymbol{\Theta}] \equiv\left\{\boldsymbol{\Theta} \mid \Theta_{i j} \in\left[\underline{\Theta}_{i j}, \bar{\Theta}_{i j}\right], \underline{\Theta}_{i j} \leq \bar{\Theta}_{i j}, \forall(i, j)\right\},
$$

the bounds of which are determined by biophysical constraints. In practice not every entry of $\Theta$ is uncertain and one seeks a representation of the Jacobian as a function solely of the uncertain vector $\boldsymbol{\theta} \in \mathbb{R}^{M}$

$$
\mathbf{J}(\boldsymbol{\theta})=\mathbf{J}_{0}+\sum_{i=1}^{M} \theta_{i} \mathbf{J}_{i}=\mathbf{J}_{0}+\boldsymbol{\Lambda} \sum_{i=1}^{M} \theta_{i} \mathbf{T}_{i}
$$

with template matrices $\mathbf{T}_{i} \in\{0,1\}^{N \times L}$. We do not exclude the case that one uncertain parameter controls multiple entries of $\Theta$. Alternatively, the parametric Jacobian may be expressed as a convex matrix polytope with

$$
\mathbf{J}(\boldsymbol{\theta}) \in \operatorname{co}\left\{\tilde{\mathbf{J}}_{1}, \ldots, \tilde{\mathbf{J}}_{K}\right\} \equiv\left\{\mathbf{J} \mid \mathbf{J}=\sum_{i=1}^{K} \alpha_{i} \tilde{\mathbf{J}}_{i}, \sum_{i=1}^{K} \alpha_{i}=1, \alpha_{i} \geq 0, i \in\{1, \ldots, K\}\right\}
$$

with $\operatorname{co}\{\cdot\}$, the convex hull. The image of the saturation hyper-rectangle $[\boldsymbol{\Theta}]$ under $\boldsymbol{\Lambda}$ is, in general, not a rectangle in the space of Jacobians and vertex points of $[\boldsymbol{\Theta}]$ can be mapped to the interior of the Jacobian polytope. Thus, we have $K \leq 2^{M}$ assuming that $L \geq N$, which is normally the case for reaction networks. 


\subsection{Guaranteed methods}

In the following, robust control methods are discussed that we consider particularly suitable for the SKM framework. They determine saturation subintervals, where stability of every member is guaranteed. The application of those methods to a model of the glycolytic pathway within the SKM framework is presented in [16].

Given a single Jacobian $\mathbf{J} \in \mathbb{R}^{N \times N}$ of a linearized dynamics, stability can be determined by checking the Hurwitz property, i.e., whether all roots of the characteristic polynomial $p(\lambda)=\operatorname{det}(\mathbf{J}-\lambda \mathbf{I})$ have negative real parts. For the case of parametric Jacobians $\mathbf{J}(\boldsymbol{\theta})$ the following theorem due to Kharitonov [15] can be utilized.

Theorem 1. Every polynomial

$$
p(\lambda, \mathbf{c})=c_{0}+c_{1} \lambda+\cdots+c_{n-1} \lambda^{n-1}+c_{n} \lambda^{n}
$$

of degree $n$ which is an instance of the polynomial set $p(\lambda,[\mathbf{c}])=\{p(\lambda, \mathbf{c}) \mid \mathbf{c} \in[\mathbf{c}]\}$ and $c_{n}>0$ is a Hurwitz polynomial, if and only if the associated following four Kharitonov polynomials

$$
\begin{aligned}
& p^{+-}(\lambda, \mathbf{c})=\bar{c}_{0}+\underline{c}_{1} \lambda+\underline{c}_{2} \lambda^{2}+\bar{c}_{3} \lambda^{3}+\bar{c}_{4} \lambda^{4}+\underline{c}_{5} \lambda^{5}+\cdots \\
& p^{++}(\lambda, \mathbf{c})=\bar{c}_{0}+\bar{c}_{1} \lambda+\underline{c}_{2} \lambda^{2}+\underline{c}_{3} \lambda^{3}+\bar{c}_{4} \lambda^{4}+\bar{c}_{5} \lambda^{5}+\cdots \\
& p^{-+}(\lambda, \mathbf{c})=\underline{c}_{0}+\bar{c}_{1} \lambda+\bar{c}_{2} \lambda^{2}+\underline{c}_{3} \lambda^{3}+\underline{c}_{4} \lambda^{4}+\bar{c}_{5} \lambda^{5}+\cdots \\
& p^{--}(\lambda, \mathbf{c})=\underline{c}_{0}+\underline{c}_{1} \lambda+\bar{c}_{2} \lambda^{2}+\bar{c}_{3} \lambda^{3}+\underline{c}_{4} \lambda^{4}+\underline{c}_{5} \lambda^{5}+\cdots
\end{aligned}
$$

are Hurwitz polynomials.

The theorem gives a necessary and sufficient condition for stability. However, the necessity is lost if the coefficients $\mathbf{c}$ are not independent as it is the case for the characteristic polynomial $p(\lambda, \boldsymbol{\theta})=\operatorname{det}(\mathbf{J}(\boldsymbol{\theta})-\lambda \mathbf{I})$. Thus, the theorem just provides a sufficient condition, and gives conservative results in general. In practice, one can obtain the overbounding coefficient intervals $[\mathbf{c}]$ by computing the characteristic polynomial with $\boldsymbol{\theta} \in[\boldsymbol{\theta}]$ using interval arithmetic $[13,16]$.

Quadratic stability of a polytopic linear system with $\mathbf{J}([\boldsymbol{\theta}])$ is defined that for each member $\mathbf{J}(\boldsymbol{\theta}) \in \operatorname{co}\left\{\tilde{\mathbf{J}}_{1}, \ldots, \tilde{\mathbf{J}}_{K}\right\}$ one can find one common quadratic Lyapunov function. With that, quadratic stability is stronger than testing the Hurwitz stability of each member. Thus, for an uncertain system that is quadratically stable all members are Hurwitz stable, but a system that is not quadratically stable can still be stable for all members. Quadratic stability thus provides just another means to obtain conservative stability bounds. However, quadratic stability, by itself, can be determined without conservatism with a finite number of tests.

Theorem 2. A linear polytopic system is quadratically stable if and only if all its vertex systems are stable.

It remains to find a common Lyapunov function for all vertex systems. This can be done by solving the following $K$ linear matrix inequalities simultaneously 


$$
\tilde{\mathbf{J}}_{i}^{T} \mathbf{P}+\mathbf{P} \tilde{\mathbf{J}}_{i} \prec \mathbf{0},
$$

for $i \in\{1, \ldots, K\}$ and $\mathbf{P} \succ 0$, the common positive-definite Lyapunov matrix. The proof of the theorem is based on the observation that any positive linear combination of negative definite terms is again negative definite

$$
\tilde{\mathbf{J}}^{T}(\boldsymbol{\alpha}) \mathbf{P}+\mathbf{P} \tilde{\mathbf{J}}(\boldsymbol{\alpha})=\sum_{i=1}^{K} \alpha_{i}\left(\tilde{\mathbf{J}}_{i}^{T} \mathbf{P}+\mathbf{P} \tilde{\mathbf{J}}_{i}\right) \prec \mathbf{0} \quad \text { with } \quad \sum_{i=1}^{K} \alpha_{i}=1 \quad \text { and } \quad \alpha_{i} \geq 0,
$$

$i \in\{1, \ldots, K\}$ and $\boldsymbol{\alpha} \equiv\left(\alpha_{1}, \ldots, \alpha_{K}\right)$.

In contrast to quadratic stability, affine quadratic stability searches for a quadratic parameter-dependent Lyapunov function, where the parameter dependency is assumed to be affine

$$
\mathbf{P}(\boldsymbol{\theta})=\mathbf{P}_{0}+\sum_{j=1}^{M} \theta_{j} \mathbf{P}_{j}
$$

Writing it in terms of polytopes we seek a Lyapunov matrix such that

$$
\tilde{\mathbf{J}}(\boldsymbol{\alpha})^{T} \tilde{\mathbf{P}}(\boldsymbol{\alpha})+\tilde{\mathbf{P}}(\boldsymbol{\alpha}) \tilde{\mathbf{J}}(\boldsymbol{\alpha}) \prec \mathbf{0}
$$

and $\tilde{\mathbf{P}}(\boldsymbol{\alpha}) \succ \mathbf{0}$ for any convex combination $\boldsymbol{\alpha}$. We used the corresponding polytopic representation of the affine set (11)

$$
\tilde{\mathbf{P}}(\boldsymbol{\alpha})=\sum_{i=1}^{K} \alpha_{i} \tilde{\mathbf{P}}_{i} \quad \text { where } \quad \sum_{k=1}^{K} \alpha_{k}=1 \quad \text { and } \quad \alpha_{k} \geq 0
$$

with the vertex matrices $\tilde{\mathbf{P}}_{i}$. Affine quadratic stability leads to bilinear matrix inequalities that are difficult to solve numerically. However, forcing another constraint on the Lyapunov function, namely multi-convexity $[2,11]$ one arrives at vertex conditions similar to the one of quadratic stability

$$
\begin{aligned}
\tilde{\mathbf{J}}_{i}^{T} \tilde{\mathbf{P}}_{i}+\tilde{\mathbf{P}}_{i} \tilde{\mathbf{J}}_{i} & \prec \mathbf{0} \\
\tilde{\mathbf{P}}_{i} & \succ \mathbf{0} \\
\mathbf{J}_{j}^{T} \mathbf{P}_{j}+\mathbf{P}_{j} \mathbf{J}_{j} & \prec \mathbf{0}
\end{aligned}
$$

for all $i \in\{1, \ldots, K\}$ and $j \in\{1, \ldots M\}$, where we used the affine representation of (7). The incorporation of multi-convexity (third inequality) introduces conservatism but yields a set of linear matrix inequalities that can now be solved efficiently using semi-definite programming.

\subsection{Efficient random sampling}

A downside of guaranteed methods of robust control is that they, in general, provide binary answers regarding stability. For instance the semidefinite program underly- 
ing quadratic stability qualifies the proposed parameter interval $[\boldsymbol{\theta}]$ as feasible or not. Thus, these methods do not lend themselves to locate the stable region or to determine the most constraining parameter dimensions. Quadratic stability can be extended to return a scalar variable, for which a proposal interval need to scaled uniformly around an expansion in order to meet quadratic stability [6]. However, also this requires a priori information about the proper expansion point and side-length ratios of the hyper-rectangle. Moreover, determination of the maximum-volume hyper-rectangle that can be inscribed into a closed surface, itself requires the solution of a nonlinear program. Multi-dimensional bisection methods is used in [16] to expand hyper-rectangles based on the binary decisions returned by the guaranteed methods of Section 3.1. However, such an approach does not scale well with the parameter dimension and also does not guarantee to converge to the maximal-volume rectangle, on top of the conservatism of those guaranteed methods.

The procedure outlined in the following aims to find a non-guaranteed hyperrectangle through advanced random sampling of the stability region. Sketching the stability region in this way, also allows one to identify parameter combinations that are most constraining in terms of stability. This can be achieved through a minor component analysis (MCA) [9]. Besides its relevance in its own rights, the obtained rectangle can then be proposed to a guaranteed method. Even if a downscaling of the rectangle is necessary due to conservatism of the guaranteed method or due to the overapproximation of the stability region by the sampling method, the expansion center and the side-lengths ratios are likely to be representative.

Sampling. We randomly sample one-dimensional information through the following theorem that provides sufficient and necessary conditions in case of onedimensional uncertainty [5].

Theorem 3. Consider the affine uncertain system $\mathbf{J}(\omega)=\mathbf{J}_{0}+\omega \mathbf{J}_{1}$, with $\mathbf{J}_{0}$ Hurwitz stable and $\omega \in[\underline{\omega}, \bar{\omega}] \in \mathbb{I} \mathbb{R}$. The matrix $\mathbf{J}(\omega)$ is robustly stable if and only if $\omega \in$ $\left[\underline{\omega}^{*}, \bar{\omega}^{*}\right]$ with

$$
\begin{aligned}
\underline{\omega}^{*} & =\frac{1}{\lambda_{\min }^{-}\left[-\left(\mathbf{J}_{0} \oplus \mathbf{J}_{0}\right)^{-1}\left(\mathbf{J}_{1} \oplus \mathbf{J}_{1}\right)\right]} \\
\bar{\omega}^{*} & =\frac{1}{\lambda_{\max }^{+}\left[-\left(\mathbf{J}_{0} \oplus \mathbf{J}_{0}\right)^{-1}\left(\mathbf{J}_{1} \oplus \mathbf{J}_{1}\right)\right]}
\end{aligned}
$$

with the Kronecker sum $\mathbf{J}_{0} \oplus \mathbf{J}_{0} \equiv \mathbf{J}_{0} \otimes \mathbf{I}_{N}+\mathbf{I}_{N} \otimes \mathbf{J}_{0}$ and with $\lambda_{\min }^{-}(\cdot)$ and $\lambda_{\max }^{+}(\cdot)$ the minimum and maximum of the strictly negative and strictly positive set of eigenvalues of a matrix.

With a nominal parameter set that corresponds to a stable Jacobian $\mathbf{J}_{0}$ the theorem provides a means to sample the stability region around this expansion point without any conservatism. We do this by shooting Bialas rays in random directions $\boldsymbol{\theta}$ from this expansion point. In vector notation this reads

$$
\operatorname{vec}(\mathbf{J}(\omega))=(\mathbf{I} \otimes \boldsymbol{\Lambda}) \operatorname{vec}(\boldsymbol{\Theta})=\operatorname{vec}\left(\mathbf{J}_{0}\right)+\omega(\mathbf{I} \otimes \boldsymbol{\Lambda}) \mathbf{R} \boldsymbol{\theta},
$$

with the appropriate rearrangement matrix $\mathbf{R} \in\{0,1\}^{L N \times M}$. The probability distribution over ray directions should be chosen such, that the intersection points between rays and stability boundary are distributed uniformly. Choosing random 
directions from an expansion point within a surface that result in a uniform distribution at that surface is a known problem; see for instance the problem of uniformly sampling the surface of a $n$-sphere [19]. However, in the absence of information on the surface to be sampled, we propose to resort to a sequential Monte-Carlo algorithm that generates a uniform distribution at a bounding rectangle $[\boldsymbol{\theta}] \subseteq[\boldsymbol{\theta}]_{0}$ that is updated during sampling. We refer to $[\boldsymbol{\theta}]_{0}$ as the outer interval determined by biophysical bounds.

Dimensionality reduction. Several expansion centers are chosen according to a tree structure with predetermined depth and degree. Parameter combinations that are constrained in terms of stability are revealed by computing an eigendecomposition of the covariance matrix of the sampled line set (see for instance Fig. 2 in Section 4). The eigendirections corresponding to small eigenvalues indicate constrained parameter combinations (minor components) [9]. Inner products between eigendirections and basis vectors of the parameter coordinates allow to identify single parameters, that are most aligned with these constrained directions. This opens up the possibility for model reduction, where interval stability is investigated only for the most constrained parameters.

Rectangle Inscription. In order to be able to inscribe a hyper-rectangle into a sampled closed surface, the samples need to be connected to give closed surface. The most natural choice is to construct the convex hull, i.e. the smallest convex set containing the sampled points. The convex hull is a convex polytope - or bounded polyhedron and thus has besides its vertex representation also a representation as a set of half-spaces (see Minkowski-Weyl theorem). We define a polyhedron $\mathscr{P}$ as

$$
\mathscr{P} \equiv\left\{\boldsymbol{\theta} \in \mathbb{R}^{M} \mid \mathbf{A} \boldsymbol{\theta} \leq \mathbf{b}, \mathbf{A} \in \mathbb{R}^{Q \times M}, \mathbf{b} \in \mathbb{R}^{Q}\right\},
$$

with $Q$ the number of half-spaces. The problem of inscribing the maximal-volume rectangle into $\mathscr{P}$ is convex and can thus be solved efficiently on polynomial time [7]. Denoting the interval of the inscribed box as $[\boldsymbol{\theta}] \in \mathbb{R}^{M}$ we can write the convex program as

$$
\begin{aligned}
& \max _{[\boldsymbol{\theta}]} \log \operatorname{det} \mathbf{W}([\boldsymbol{\theta}]) \\
& \text { subject to } \\
& {[\boldsymbol{\theta}] \subseteq \mathscr{P},}
\end{aligned}
$$

with the diagonal matrix $\mathbf{W}(\cdot)$ denoting the interval width $\mathbf{W}([\boldsymbol{\theta}])=\operatorname{diag}\{\sup ([\boldsymbol{\theta}])-$ $\inf ([\boldsymbol{\theta}])\}$. Instead of having $2^{M}$ linear inequalities for every vertex of $[\boldsymbol{\theta}]$, the constraint $[\boldsymbol{\theta}] \subseteq \mathscr{P}$ can be expressed more efficiently with $2 M$ inequalities [7]. The plausibility of the obtained optimal $[\boldsymbol{\theta}]$ rest upon the assumption that the intersection of stability region and biophysical bounding box $[\boldsymbol{\theta}]_{0}$ can well be encoded through a convex polytope.

Utilizing the exact convex hull, i.e. the tightest convex enclosure, introduces scalability issues in high dimensions. The worst case complexity of an optimal convex hull algorithm was shown to be $\mathscr{O}\left(n^{\lfloor M / 2\rfloor}\right)$ for $M \geq 4$, where $n$ is the number of sample points. However, the worst-case is rarely encountered and the actual complexity depends on the number of necessary inequalities $Q$, the order of which can 
vary from $\mathscr{O}(1)$ to $\mathscr{O}\left(n^{\lfloor M / 2\rfloor}\right)$. Taking $Q$ into account, a polynomial algorithm in $n, M$ and $Q$ was shown to exist for the non-degenerate case [3].

\section{Application}

We apply the proposed sampling method of Section 3.2 to a medium-scale model of glycolysis, depicted in Fig. 1. Transformed to the SKM representation of Section 2, the model has 12 non-zero entries in the saturation matrix $\Theta$. For all reactions, except one, we assume standard Michaelis-Menten kinetics giving rise to biophysical bounds $\left[\theta_{i}\right]_{0}=[0,1]$ for $i \in\{2, \ldots, 12\}$. For the first reaction, the conversion of Glucose (Glc) into fructose-1,6-biphosphate (FBP) we implement the known inhibitory effect of ATP, resulting in $\left[\theta_{1}\right]_{0}=[-3,1]$. We sketch the feasible region charac-

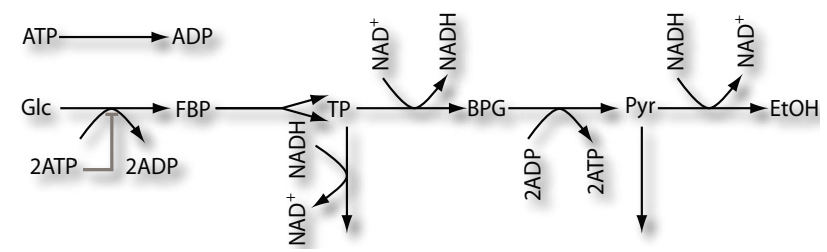

Fig. 1 Medium-scale model of the yeast glycolytic pathway comprising 8 reactions giving rise to 12 saturation parameters in the framework of structural kinetic modeling.

terized as the intersection of the stability domain with the biophysical bounding box using $10^{4}$ Bialas rays with a flat tree configuration of depth one and degree 100 . Figure 2(a) shows the eigendecomposition of the covariance matrix $\mathbf{C}$ in normalized coordinates, indicating one tightly constrained parameter combination. Inner product computation reveals that direction $\theta_{1}$ is strongly aligned with the corresponding eigendirection.

To illustrate the method we perform a model reduction retaining only the seven most constrained parameter dimensions as interval variables and adjusting the remaining ones to their nominal value, chosen to be the midpoint of $[\boldsymbol{\theta}]_{0}$. The obtained stability interval are depicted in Fig. 2(b). The convex hull and the obtained 7dimensional stability rectangle, down-projected onto the first three most constrained parameter dimension is shown in Fig. 3.

\section{Conclusions}

The scarcity of kinetic information for metabolic reactions rarely allows for the determination of detailed kinetic rate laws for a metabolic model. We combine the local stability analysis of structural kinetic modeling with interval methods to compute guaranteed and non-guaranteed stability intervals for the saturation levels of 

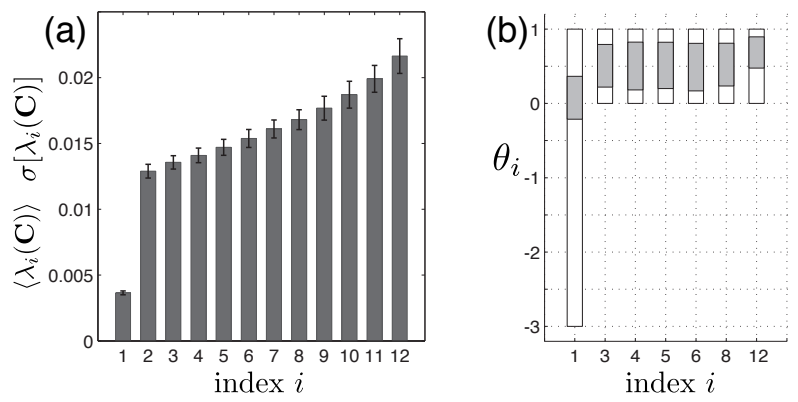

Fig. 2 (a) Mean and standard deviation of the spectrum for the covariance matrix $\mathbf{C}$ of the stable 12-dimensional parameter region - based on 100 runs each with $10^{4}$ Bialas rays (tree of depths one and degree 100). (b) biophysical bounding boxes and obtained stable intervals (gray) for parameters retained after model reduction.

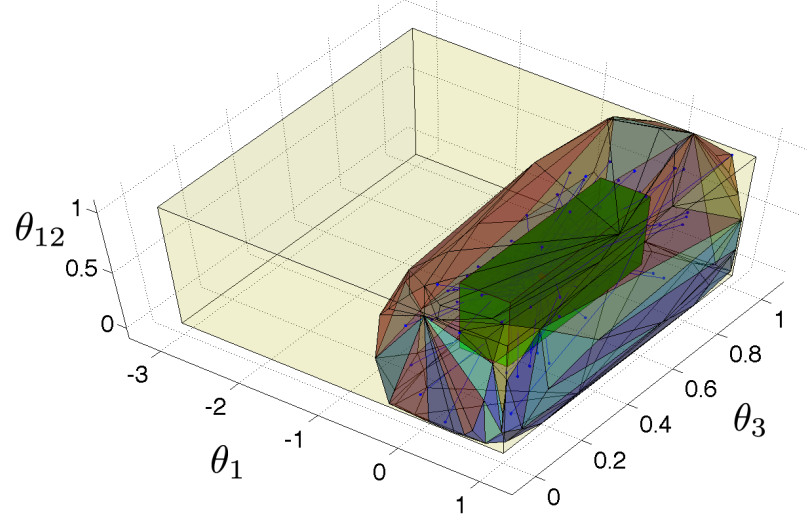

Fig. 3 Maximum-volume hyper-rectangle (green) inscribed in the convex hull (brown) and biophysical bounding box $[\boldsymbol{\theta}]_{0}$ (yellow); a few of the $10^{4}$ rays (blue) used to sketch the stability region of the reduced 7-dimensional interval system; down-projection to coordinates that are the most aligned to the directions of the first three minor components.

the involved reactions. We provide an efficient sampling algorithm to sketch highdimensional stability regions and apply methods from statistics and computational geometry to obtain non-guaranteed stability intervals. The computed stability interval may serve as a proposal for the binary test of guaranteed methods from robust control. To alleviate scalability issues in the applied computational geometry methods, one may resort to randomized algorithms, for instance such as the randomized incremental construction of the convex hull [8].

Acknowledgements HK acknowledges the support from the Swiss National Science Foundation, grant no. 200020-117975/1. SA was supported by the Laboratory of Nonlinear Systems, EPFL 
within the Summer@EPFL internship program. RS is supported by the grant FORSYS-Partner: Systems biology of cyanobacterial biofuel production, as well as by the rearch initiative SysMO: MOSES (Grant Reference: BBF0035281) and SulfoSys (Grant Reference: BBF0035361).

\section{References}

1. Ackermann, J., Bartlett, A., Kaesbauer, D., Sienel, W., Steinhauser, W.: Robust Control: Systems with Uncertain Physical Parameters. Springer-Verlag, New York, USA (2001)

2. Apkarian, P., Tuan, H.D.: Parametrized LMIs in control theory. SIAM J. Control Optim. 38(4), 1241-1264 (2000)

3. Avis, D., Bremner, D., Seidel, R.: How good are convex hull algorithms. Comput. Geom. Th. Appl. 7, 265-302 (1997)

4. Barmish, B.R.: New Tools for Robustness of Linear Systems. Macmillan Publishing Company (1994)

5. Bialas, S.: A necessary and sufficient condition for the stability of convex combinations of stable polynomials or matrices. Bull. Pol. Acad. Sci. 33, 473-480 (1985)

6. Boyd, S., El Ghaoui, L., Feron, E., Balakrishnan, V.: Linear Matrix Inequalities in System and Control Theory. SIAM, Philadelphia, USA (1994)

7. Boyd, S., Vandenberghe, L.: Convex Optimization. Cambridge University Press (2004)

8. Clarkson, K.L., Mehlhorn, K., Seidel, R.: Four results on randomized incremental construction. Comput. Geom. 3(4), 185-212 (1993)

9. Duda, R.O., Hart, P.E., Stork, D.G.: Pattern Classification. Wiley-Interscience Publication (2000)

10. Feist, A.M., Herrgard, M.J., Reed, J.L., Palsson, B.O.: Reconstruction of biochemical networks in microorganisms. Nat. Rev. Microbiol. 7(2), 129-143 (2009)

11. Gahinet, P., Apkarian, P., Chilali, M.: Affine parameter-dependent Lyapunov functions and real parametric uncertainty. IEEE Trans. Autom. Control 41(3), 436-442 (1996)

12. Heinrich, R., Schuster, S.: The regulation of cellular systems. Chapman Hall, New York (1996)

13. Jaulin, L., Kieffer, M., Didrit, O., Walter, E.: Applied Interval Analysis. Springer, London,UK (2001)

14. Kell, D.B.: Metabolomics and systems biology: making sense of the soup. Curr. Opin. Microbiol. 7(3), 296-307 (2004)

15. Kharitonov, V.L.: Asymptotic stability of an equilibrium position of a family of systems of linear differential equations. Differential Equations 14, 1483-1485 (1979)

16. Koeppl, H., Hafner, M., Steuer, R.: Semi-quantitative stability analysis constrains saturation levels in metabolic networks. In: Proc. Int. Workshop on Comput. Syst. Biol., pp. 91-94. Aarhus, Denmark (2009)

17. Kruger, N.J., Ratcliffe, R.G.: Insights into plant metabolic networks from steady-state metabolic flux analysis. Biochimie 91(6), 697-702 (2009)

18. Kuiper, B.J.: Qualitative Reasoning: Modeling and Simulation with Incomplete Knowledge. MIT Press, Cambridge, MA, USA (1994)

19. Marsaglia, G.: Choosing a point from the surface of a sphere. Ann. Math. Stat. 43, 645-646 (1985)

20. Schaber, J., Liebermeister, W., Klipp, E.: Nested uncertainties in biochemical models. IET Syst. Biol. 3(1), 1-9 (2009)

21. Steuer, R., Gross, T., Balsius, B.: Structural kinetic modeling of metabolic networks. Proc. Nat. Acad. Sci. U.S.A. 103(32), 11,868-11,873 (2006)

22. Steuer, R., Junker, B.H.: Computational models of metabolism: Stability and regulation in metabolic networks. Adv. Chem. Phys. 142 (2009)

23. Wang, L., Hatzimanikatis, V.: Metabolic engineering under uncertainty. I: framework development. Metab. Eng. 8(2), 133-141 (2006)

24. Zamboni, N., Sauer, U.: Novel biological insights through metabolomics and 13c-flux analysis. Curr. Opin. Microbiol. 12(5), 553-558 (2009) 